\title{
Design and Development of a Miniaturized Embedded UHF RFID Tag for Automotive Tire Applications
}

\author{
S. Basat ${ }^{(1)}$, M.M. Tentzeris ${ }^{(1)}$, J. Laskar $^{(1)}$ \\ (1) School of ECE, Georgia Institute of Technology \\ 85 Fifth Street, $N W$ \\ Atlanta, GA 30308, USA \\ Email:gte309k@mail.gatech.edu
}

\begin{abstract}
The utilization of UHF Radio Frequency IDentifcation (RFID) tag to monitor the tire history in automotive tire applications is presented. Three novel antenna designs are proposed and comparatively evaluated in terms of efficiency, size, read range limitations, and antenna-IC port matching. A prototype of a low-resistive high-inductive RFID tag antenna that is physically built and embedded in actual tires meets the design specifications of read range of minimum $50 \mathrm{~cm}$, high-reliability in terms of the tire traveling test, and antenna-IC packaging size requirement of $7 \mathrm{~cm} \times 3 \mathrm{~cm}$.
\end{abstract}

\section{INTRODUCTION}

The recent advances in cost-effective low-power electronics and packaging have enabled the RFID tag as a likely substitute for barcodes [1] in industries such as access control, parcel and document tracking, distribution logistics, automotive systems, and livestock or pet tracking. In these applications data are contact-free transferred to a local querying system (reader or interrogator) from a remote transponder (tag) including an antenna and a microchip transmitter. A suitable antenna for these tags must have low cost, low profile and especially small size whereas the bandwidth requirement (few kilohertz) is less critical [2]. The RFID tags also present challenges in behavioral modeling and simulation of the antenna and module/package integration in parameters such as the pad capacitance, the estimation of the parasitics due to the proximity of IC and antenna, and the identification of a low-cost low-loss light material.

In this paper, three novel miniaturized antennas are presented for $915 \mathrm{MHz}$ passive tags that are designed to be embedded inside commercial automobile tires. The necessary power required to energize and activate the tag's microchip is drawn from the electromagnetic field provided by the reader unit's antenna. The transponder IC stores the tire's unique ID, which can be associated with the vehicle identification number. The chip also stores information about when and where the tire was made, its maximum inflation pressure, size and so on. The tag utilizes the low cost lead frame based IC packaging process and the miniaturized antenna is built in the lead frame.

Passive ICs are intrinsically highly reactive because of the necessary power to bias the IC which is delivered by charging up the IC through electromagnetic coupling. Due to the low resistive yet high capacitive impedance of the microchip, novel design approach for the RFID antennas have to be proposed comprising of antennas that are lowly resistive (high efficiency) and highly inductive for matching to the input impedance of the transponder IC.

An RFID tag of UHF band employs far-field radiation of the real power contained in free-space propagating electromagnetic plane waves due to its shorter wavelength, while a $13.56 \mathrm{MHz} \mathrm{HF}$ tags is utilizing inductive coupling in the near-field region as the wavelength is much longer. The IE3D design tool, which is based on method of moments $(\mathrm{MoM})$, is used to optimize and analyze the tag. This tool is used as main platform to design and come up with certain antenna performance parameters such as gain, radiation pattern, and efficiency. Three different $7 \mathrm{~cm} \times 3 \mathrm{~cm}$ (equal in area or smaller) antenna designs are built to make sure maximum range is obtained. Achieving that range has been a challenge because the rubber makes it harder to get an impedance matching. The tested tags in actual tires yield maximum operational distance ranging from $48.7 \mathrm{~cm}$ to $52.5 \mathrm{~cm}$ which is well within the required range $(50 \mathrm{~cm})$ for the application.

\section{DESIGN APPROACH}

The IC input impedance for the tire application is $17-\mathrm{j} 350 \Omega$, which means the load antenna impedance should be $17+\mathrm{j} 350 \Omega$ for maximum power transfer (conjugate matching). This requires the antenna impedance to be low- resistive yet high-inductive. Various antenna designs like dipole, printed patch, log-spiral, and meander-line have been proposed as a solution in the past. $[3,4,5,6]$ Nevertheless, a novel approach has to be followed to keep the antenna size small and 
the load impedance to have a low real part (small resistance) and a high positive imaginary part (high inductance). To achieve this, an inductive element needs to be incorporated into the antenna. In addition to this, the metal size is desired to be as big as possible to obtain better radiation parameters such as directivity and efficiency through the larger radiating aperture, though it could increase the metal loss leading to a trade-off in the antenna efficiency. Increasing the metal size also lowers the surface resistance and increases current flow as shown in Fig. 1. It is for these abovementioned reasons, a dipole antenna with inductive stubs and a metal patch is used as the basis for the three antenna designs of this paper. The stubs provide the inductive load impedance meanwhile the metal patch lowers the load resistance.

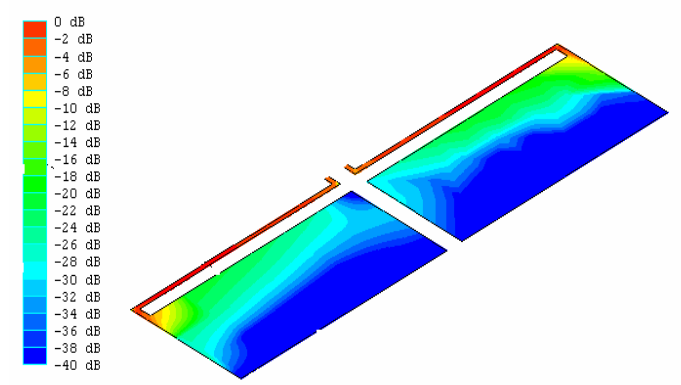

Fig. 1 Current flow in UHF RFID Tag antenna

To accomplish maximum directivity and optimum radiation, the designs are built to achieve half-wavelength $\left(\lambda_{\mathrm{r}} / 2\right.$ $\sim 9 \mathrm{~cm}$ in rubber material @ $915 \mathrm{MHz}$ ) resonance at first. In essence, the designs possess similarity to the half-wave dipole antenna; however, there exists a trade-off between antenna-IC matching and resonance. Whenever the size is increased to match for resonance, antenna-IC matching deteriorates. The miniaturization of the antenna size is another issue, which requires the length of the antenna to be smaller than the resonance length.

\section{ANTENNA DESIGN}

The three RFID Tag antenna designs are shown in Fig. 2. These antennas are made of copper metal with thickness of $200 \mathrm{um}$. The antenna is embedded inside tire material that is basically rubber. $\left(\varepsilon_{\mathrm{r}}=3.0, \tan \delta=0.02\right)$ In addition to this,

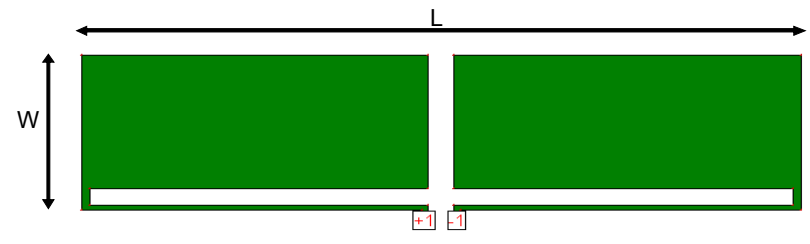

(a) Antenna\#1 where minimum line spacing is $1.5 \mathrm{~mm}$ with $\mathrm{W}=14.5 \mathrm{~mm}, \mathrm{~L}=56 \mathrm{~mm}, 2 \mathrm{~mm}$ port separation, and trace width of $0.5 \mathrm{~mm}$

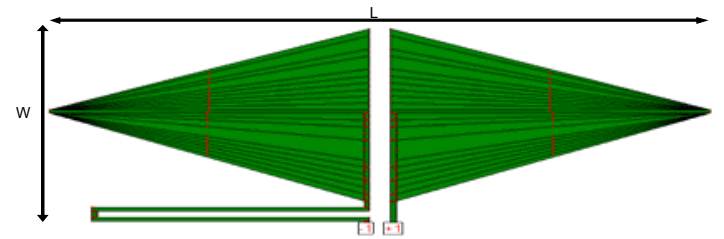

(b) Antenna\#2 where minimum line spacing is $0.5 \mathrm{~mm}$ with $\mathrm{W}=30 \mathrm{~mm}, \mathrm{~L}=60 \mathrm{~mm}, 2 \mathrm{~mm}$ port separation and trace width of $0.5 \mathrm{~mm}$.

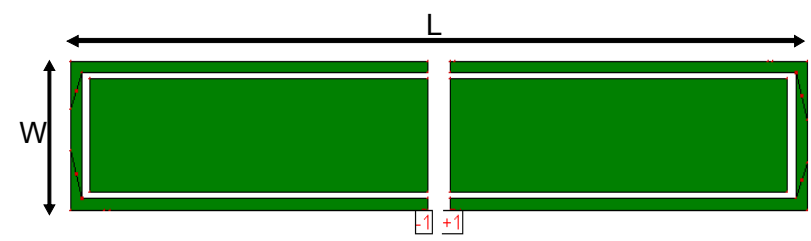

(c) Antenna\#3 where minimum line spacing is $0.5 \mathrm{~mm}$ with $\mathrm{W}=12 \mathrm{~mm}, \mathrm{~L}=67 \mathrm{~mm}, 2 \mathrm{~mm}$ port separation and trace width of $0.5 \mathrm{~mm}$.

Fig. 2 The three different RFID antenna designs for tire application 
one-port differential excitation, which is used to measure the actual antenna-IC configuration, is employed to numerically calculate the return loss and antenna load impedance as well as the read range measurement.

The single inductor stub as shown in Fig. $2 \mathrm{~b}$ is utilized to obtain the required inductance where the triangular patch is the main radiator for Antenna\#2. The other two designs utilize double stub configurations. Antenna\#1 and Antenna\#3 as shown in Fig. 2a, c are also highly inductive due to the double stubs that are easily incorporated into the radiator rectangular patches. This feature is proven to be very important to enhance radiation because the inductive stub, which is used for antenna-IC reactance matching, becomes more part of the radiating element.

\section{ANTENNA RESULTS AND DISCUSSION}

The radiation patterns for the three designs are shown in Fig. 3. All of the designs are omni-directional in the vertical plane which is actually desired because maximum radiation should be achieved when the tag is read in the plane that is perpendicular to the RFID antenna. The horizontal radiation is also crucial in terms of the orientation of the reader. The Interrogator (reader) does not necessarily have to be positioned on top or bottom with respect to the RFID tag, but the tire RFID tag should also have the functionality to be read from the sides as well. For this reason, the second antenna is designed to overcome this obstacle in case the application requires effective radiation characteristics in the azimuth (horizontal) plane. This is demonstrated in Fig. $3 \mathrm{~b}$ where the radiation patterns are almost identical for both planes.

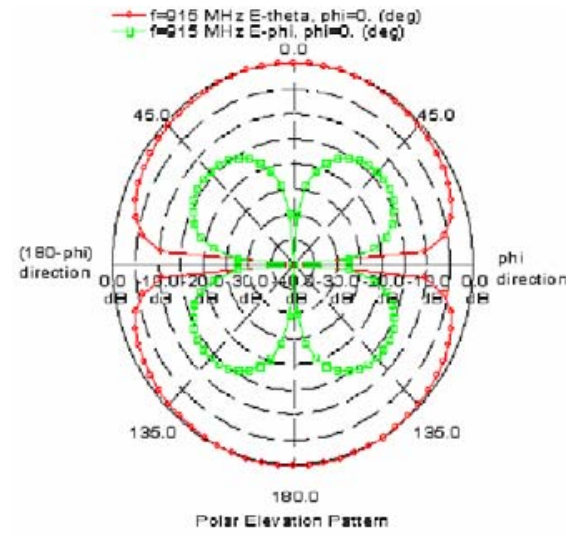

(a) antenna\#1

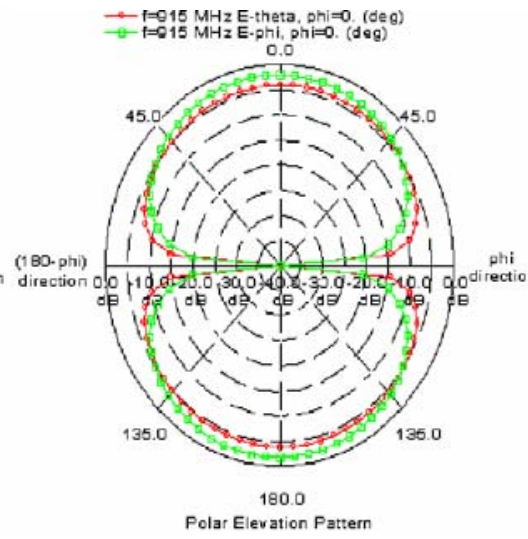

(b) antenna\#2

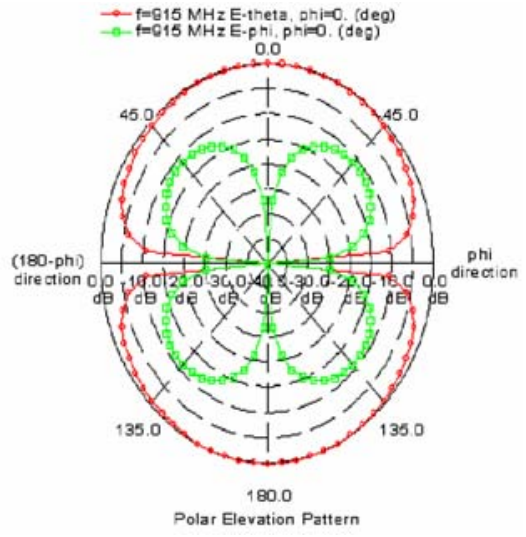

(c) antenna\#3

Fig. 3 Horizontal (E-phi) and vertical (E-theta) plane radiation patterns for the three antenna designs.

Antennas are located in the horizontal plane.

The antenna load impedances for the three antennas are shown in Fig. 4, verifying that they are high-inductive

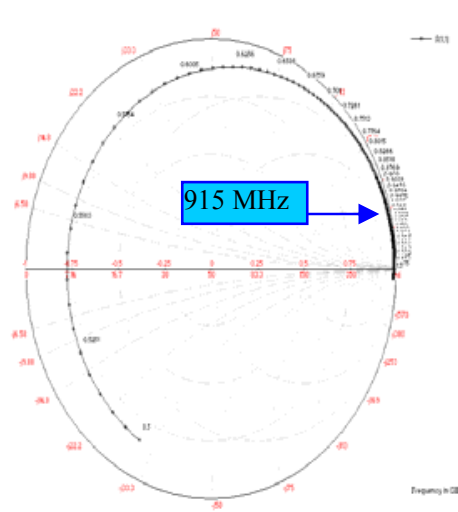

(a) antenna\#1

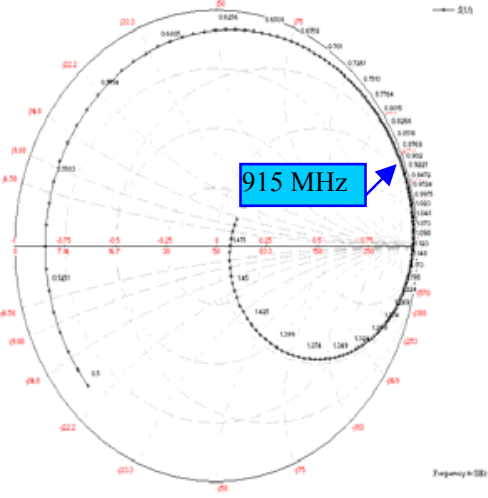

(b) antenna\#2

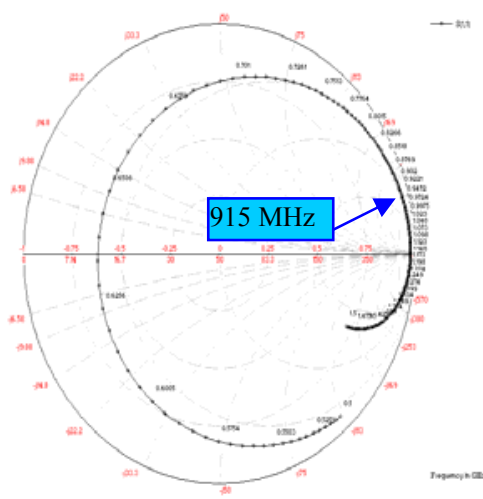

(c) antenna\#3

Fig. $4 S_{11}$ input load impedance Smith chart plots for the three antenna designs (range of frequency $=500-1500$ MHz). 
and low-resistive. The load impedance values are displayed in Table 1 along with other radiation parameters namely

Table 1. Simulated antenna parameters and measured read range.

\begin{tabular}{|c|c|c|c|c|c|c|}
\hline & Imp & $\begin{array}{c}\mathrm{RL} \\
(\mathrm{dB})\end{array}$ & $\mathrm{D}(\mathrm{dBi})$ & Eff.(9) & $\begin{array}{l}\text { Range(cm) } \\
\text { (in tire } \\
\text { material) }\end{array}$ & $\begin{array}{l}\text { Range(cm) } \\
\text { (lin actual } \\
\text { tire) }\end{array}$ \\
\hline Ant茾1 & $\begin{array}{l}16.6+ \\
1282.7\end{array}$ & -19.47 & 2.81 & 7.79 & 125 & 52.8 \\
\hline Ant\#2 & $\begin{array}{c}178+ \\
j 318.1\end{array}$ & -26.44 & 2.85 & 5.43 & 80 & 48.7 \\
\hline AntH3 & $\begin{array}{l}44.9+ \\
j 331.7\end{array}$ & -26.24 & 2.72 & 6.75 & 125 & 52 \\
\hline
\end{tabular}

return loss, directivity, radiation efficiency, and read range in tire material and in actual tire. As it can be seen from Table 1, the antenna efficiency determines how much operational distance is needed for the tag. For example, although Antenna\#2 and Antenna\#3 exhibit almost the same return loss and directivity, the read range is higher for Antenna\#3 due to higher radiation efficiency of this antenna. Still, the read range does not only depend on the efficiency of the radiating antenna.

The first set of read ranges as presented in Table 1 are measured only with RFID tag in tire material. When the tag is embedded in actual commercial tires, Antenna\#1,\#2, and \#3 yield operational distances of $52.8,48.7,52.0 \mathrm{~cm}$ respectively. The application requires a read range of $50 \mathrm{~cm}$ due to anti-collision limitations, so the three designs are acceptable including antenna\#2 which requires further minor optimization. The reason for the read range reduction is the presence of the steel meshes on the inner and outer surfaces of the tire.

Further efficiency improvement can be accomplished in three ways: 1) Create more coupling by surrounding the radiating patch with the inductive stub. The stubs that enclose or join with the patch generate more current flow which enables the antenna to radiate more. 2) Make the inductive stub more integrated with the radiating patch by joining the stub and the patch. 3) Use a less lossy dielectric material which would minimize excitation of substrate modes and power leakage into the dielectric.

\section{CONCLUSIONS}

Three novel antennas have been proposed for $915 \mathrm{MHz}$ UHF RFID applications for tires. These antennas with low resistance and high inductance for the input impedance provide a good example of a design procedure if the load impedance from the transponder is unusually high in capacitance and low in resistance. The tag size plays a major role in determining the read range: The smaller the tag, the smaller the energy capture area, therefore the shorter the read range, especially complicated lossy media such as tires. A proper design of the system and a thorough optimization of the interrogator power, the antenna positioning and orientation, and an optimum tag in-tire positioning helps to alleviate this limitation. It has been observed that the effective read range also depends on the absorption/attenuation factor of the type of the material in which the tag is embedded.

\section{REFERENCES}

1. G. Prophet, "RFID and the smart label; bye-bye bar code?", EDN-Europe, pp.26-36, June 2000.

2. Vince Stanford, "Pervasive computing goes the last hundred feet with RFID systems", IEEE CS \& IEEE ComSoc, 2003.

3. Gaetano Marrocco, Alessandro Fonte, and Fernando Bardati,"Evolutionary design of miniaturized meander-line antennas for RFID applications", IEEE Trans. Antennas \&Propagaion, pp.362-365, 2002.

4. Gaetano Marrocco, "Gain-optimized self-resonant meander-line antennas for RFID applications", IEEE Antennas \& Wireless Propagation Letters, Vol. 2, pp.302-305, 2003.

5. P.R. Foster and R.A. Burberry," Antenna problems in RFID systems", Microwave and Antenna Systems, IEE,Vol. 3, pp.1-5, 1999

6. Xianming Qing and Ning Yang, “A folded dipole antenna for RFID”, IEEE Antennas \& Propagaion Conference, 2004. 